M. Comte · J. I. Díaz

\title{
On the Newton partially flat minimal resistance body type problems
}

Received December 12, 2004

\begin{abstract}
We study the flat region of stationary points of the functional $\int_{\Omega} F(|\nabla u(x)|) d x$ under the constraint $u \leq M$, where $\Omega$ is a bounded domain in $\mathbb{R}^{2}$. Here $F(s)$ is a function which is concave for $s$ small and convex for $s$ large, and $M>0$ is a given constant. The problem generalizes the classical minimal resistance body problems considered by Newton. We construct a family of partially flat radial solutions to the associated stationary problem when $\Omega$ is a ball. We also analyze some other qualitative properties. Moreover, we show the uniqueness of a radial solution minimizing the above mentioned functional. Finally, we consider nonsymmetric domains $\Omega$ and provide sufficient conditions which ensure that a stationary solution has a flat part.
\end{abstract}

Keywords. Newton problem, obstacle problem, quasilinear elliptic operators, flat solutions

\section{Introduction}

Seventy years prior to the derivation of the conservation laws for a nonviscous compressible fluid by L. Euler in 1755 ([10]), I. Newton introduced, in 1685, one of the pioneering problems in the Calculus of Variations: find the shape of a symmetrical revolution body moving in a fluid with minimal resistance to motion (see [16]). As a matter of fact, the problem had already been suggested by Galileo in his famous Discursi in 1638 (for a detailed history see Goldstine [11]). Newton was able to derive the resistance law of the body under the following assumptions. Firstly, he supposed that particles do not interact with each other, "a rare medium consisting of equal particles freely disposed at equal distances" 1 , and that each particle impacts the body at most once. Secondly, the impacts were assumed to be elastic and the resistance proportional to the impact angle. If we write the problem in terms of a vertical flow, we can describe the body as $B=\{(x, z): x \in \Omega$, $0 \leq z \leq u(x)\}$, with $u(x)=0$ for $x \in \partial \Omega$ and for a given bottom set $\Omega$ in $\mathbb{R}^{2}$. In this

Research of J. I. Díaz partially supported by project MTM2004-07590 of the DGISGPI (Spain). RTN 2002-00274 "Fronts and singularities" of the European Union.

M. Comte: Université Pierre et Marie Curie, Laboratoire Jacques-Louis Lions, 75252 Paris Cedex 05, France; e-mail: comte@ann.jussieu.fr; web: www.ann.jussieu.fr

J. I. Díaz: Departamento de Matematica Aplicada, Universidad Complutense de Madrid, Madrid 28040, Spain; e-mail: ji_diaz@mat.ucm.es; web: www.mat.ucm.es/ jidiaz

Mathematics Subject Classification (2000): Primary 49J40; Secondary 35R35

1 In Newton's original formulation. 
framework it is not too difficult to show (see, for instance, [1], [6] and [17]) that the total resistance of the body is proportional to the integral

$$
I(u)=\int_{\Omega} \frac{1}{1+|\nabla u(x)|^{2}} d x .
$$

As mentioned in Armanini ([1]), in the same historical book Newton also considered other resistance assumptions leading to different power expressions of the type

$$
\int_{\Omega} \frac{1}{1+|\nabla u(x)|^{n}} d x
$$

with $n \geq 1$.

In order to guarantee a single impact, it is common to assume the body to be concave. Nevertheless, some other profiles have been considered in the literature for the more general case in which any particle hitting the graph of $u$ with vertical velocity does not hit again (see [8]).

If some of Newton's original assumptions are weakened, one might derive a similar functional, by adding a correction term. For instance, in $[17]^{2}$ a resistance functional of the type

$$
\int_{\Omega} \frac{1}{1+|\nabla u(x)|^{n}} d x+\int_{\Omega} p(x, u(x)) d x
$$

was proposed. Nevertheless, it is worth mentioning that even though Newton's resistance model is only a crude approximation, it appears to provide good results in many contexts, for instance, when dealing with a rarified gas in hypersonic aerodynamics. Many distinguished specialists in this area, von Karman, Ferrari, Lightill, and Sears have used this model (see the exposition in the NASA report [9] and the book [15]).

In Newton's formulation one looks for a minimum of the functional (1) (or (2)) in the class of (suitably regular) functions satisfying two unilateral conditions $0 \leq u(x) \leq M$ for $x \in \Omega$. Due to that fact, the associated Euler-Lagrange equations must be suitably understood, for instance, in terms of some variational inequality (for a general exposition of this theory see, for instance, [13]). It can be shown that the Lagrange multiplier term associated with the unilateral condition $0 \leq u$ vanishes due to the fact that the special form of the functional leads to the concavity of any possible stationary point $u(x)$ of that functional, and thus this unilateral condition is trivially satisfied once we assume the other (and crucial) unilateral condition $u \leq M$ (which, to the contrary, leads to a nonvanishing Lagrange multiplier term).

It is worth mentioning that the integrand in (1) (and (2p) is not globally convex in $\nabla u$ (although it is a convex function when $|\nabla u(x)| \geq \alpha$ for some suitable $\alpha>0$ ). Moreover it is not coercive (in fact it converges to zero as $|\nabla u(x)| \rightarrow+\infty$ ). Those two facts arise quite often in many other special (but relevant) problems of the Calculus of Variations (see, for instance, some other classical and more recent examples mentioned in [2]).

\footnotetext{
2 Here Wagner allows multiple impacts.
} 
This motivates us to consider a general class of functionals (invariant under symmetrical changes of coordinates) of the form

$$
\int_{\Omega} F(|\nabla u(x)|) d x
$$

with

$$
F(|\nabla u|) \rightarrow 0 \quad \text { as }|\nabla u| \rightarrow+\infty
$$

(the case of integrands of the form $F(|\nabla u(x)|)-f(x) u(x)$ with $f(x) \neq 0$ will be the subject of a separate study [7]). Actually, we shall not deal with the associated minimization problem, but with the more general case of the Euler-Lagrange variational inequality satisfied by any stationary point $u$ fulfilling the unilateral constraint $u \leq M$. So, given $M>0$, we shall consider a class of quasilinear obstacle problems which can be formulated as follows:

$$
\text { (OP) } \begin{cases}-\operatorname{div}(A(|\nabla u|) \nabla u)+\beta(u) \ni 0 & \text { in } \Omega, \\ u=0 & \text { on } \partial \Omega,\end{cases}
$$

where $\beta$ is the maximal monotone graph (see Brezis [3]) in $\mathbb{R}^{2}$ given by

$$
\begin{cases}\beta(u)=\{0\} & \text { if } u<M, \\ \beta(M)=[0,+\infty), & \\ \beta(u)=\emptyset & \text { if } u>M,\end{cases}
$$

and $A \in C^{1}(0,+\infty)$ satisfies the following set of assumptions: there exists $\alpha_{A} \geq 0$ such that

the function $t \mapsto t A(t)$ is decreasing on $\left(0, \alpha_{A}\right)$ and increasing on $\left(\alpha_{A},+\infty\right)$,

$$
\begin{gathered}
A<0 \quad \text { on }[0,+\infty) \text { and } \lim _{t \rightarrow+\infty} t A(t)=0, \\
\lim _{a \rightarrow+\infty} A(a) \int_{\alpha_{A}}^{a} \frac{d \tau}{\tau A(\tau)}<1 .
\end{gathered}
$$

In order to establish the existence of solutions for this type of noncoercive problems, several different additional conditions have been introduced in the literature (mainly the concavity of $u$ : see [5] and its list of references). In this paper we shall deal with solutions of the obstacle problem (OP) in the class of functions such that

$$
u \in H_{0}^{1}(\Omega) \text { and }|\nabla u(x)| \geq \alpha_{A} \quad \text { if } u(x)<M .
$$

We first consider the radial case corresponding to $\Omega=B(0, R)$ and $u$ in the class of radially symmetric functions satisfying (9). It is easy to see that then the problem reduces to the study of the one-dimensional free boundary value problem

$$
\left\{\begin{array}{l}
-\frac{1}{r} \frac{d}{d r}\left(r A\left(\left|u^{\prime}\right|\right) u^{\prime}\right)=0 \quad \text { in }(\rho, R), \\
u(R)=0, \quad u(\rho)=M \\
-u^{\prime}\left(R^{-}\right)>\alpha_{A}
\end{array}\right.
$$

where $\rho \in[0, R)$ must be determined. Notice that the function $u$ extended by $M$ to $\Omega$ satisfies (OP). Our main result is 
Theorem 1.1. Let $R>0$ be given. Assume that A satisfies the above conditions. Then, for every $M>0$, there exists $\rho_{M} \in(0, R)$ such that for any $m \in\left(\alpha_{A},+\infty\right)$ there exists a solution $u(r)=u(r ; m)$ of the obstacle problem which satisfies

(i) $u \equiv M$ in $\left[0, \rho_{m}\right]$ for some $\rho_{m} \in\left[\rho_{M}, R\right)$,

(ii) $-u^{\prime}\left(\rho_{m}\right)=m$,

(iii) $u$ is strictly concave in $\left(\rho_{m}, R\right)$ and $u \in W^{1, \infty}(0, R)$.

Finally, the map $M \mapsto \rho_{M}$ is decreasing and convex.

We prove this theorem in Section 3, even under more general conditions on $A$.

Moreover, we prove that although there is not a unique solution to problem (10), there is a unique radial minimizer for the functional (3) in the class of solutions of the associated problem (10).

In the Newton case, P. Guasoni established in [12] the existence of a function in $H_{0}^{1}(\Omega)$ which is not radially symmetric, for which the value of the functional is smaller than any value arising from a radial function. On the other hand, T. Lachand-Robert and É. Oudet found in [14] numerically another function leading to an even smaller value of the functional. We conjecture that similarly, radial solutions are not minimizers either for the more general class of functions $F$ considered in this paper.

Another consequence of our results is that they reveal some kind of optimality of the structure assumptions made in the regularity result by H. Brezis and D. Kinderlehrer. Indeed, these authors established in [4] that, if the quasilinear operator $A$ is "locally coercive", the solution of the associated obstacle problem fulfils $u \in W^{2, s}$ for every $1<s<+\infty$. In contrast, we show here that the solutions of (OP) are not of class $C^{1}$.

Finally, a study of the coincidence set (the flat region of the body) without any symmetry assumption is presented in Section 4. In particular, we obtain some answers to a question raised in [6, question (vi) on page 11].

\section{Statement of the problem}

We consider the problem

$$
\left\{\begin{array}{l}
-\operatorname{div}(A(|\nabla u|) \nabla u)+\beta(u) \ni 0 \quad \text { in } \Omega, \\
u \in H_{0}^{1}(\Omega), \quad|\nabla u| \geq \alpha_{A} \text { if } u<M,
\end{array}\right.
$$

where $\beta$ is the maximal monotone graph in $\mathbb{R}^{2}$ given by

$$
\begin{cases}\beta(u)=\{0\} & \text { if } u<M, \\ \beta(M)=[0,+\infty), & \\ \beta(u)=\emptyset & \text { if } u>M,\end{cases}
$$

and

$$
A \in C^{1}(0,+\infty)
$$

is such that there exists $\alpha_{A} \geq 0$ for which

$$
\text { the function } t \mapsto t A(t) \text { is decreasing on }\left(0, \alpha_{A}\right) \text {. }
$$


We also assume one the following sets of conditions: either

$$
\begin{gathered}
t \mapsto t A(t) \text { is increasing on }\left(\alpha_{A},+\infty\right), \\
A<0 \quad \text { on }[0,+\infty), \quad \lim _{t \rightarrow+\infty} t A(t)=0, \\
\lim _{a \rightarrow+\infty} A(a) \int_{\alpha_{A}}^{a} \frac{d \tau}{\tau A(\tau)}<1,
\end{gathered}
$$

or there exists $\beta_{A} \geq \alpha_{A} \geq 0$ such that

$$
\begin{gathered}
t \mapsto t A(t) \text { is increasing on }\left(\alpha_{A}, \beta_{A}\right), \\
A<0 \quad \text { on }\left[0, \beta_{A}\right), \quad A\left(\beta_{A}\right)=0, \\
\int^{\beta^{-}} \frac{d \tau}{\tau|A(\tau)|}<+\infty .
\end{gathered}
$$

Throughout, we will refer to assumptions (12), (13), (14), (15) and (16) as Case 1, and assumptions (12), 13, (17), 18) and (19) as Case 2.

Example 1. In the classical Newton obstacle problem, we search

$$
\operatorname{Min}_{K} \int_{\Omega} F(|\nabla u|) d x, \quad F(t)=\left(1+t^{2}\right)^{-1},
$$

where $K=\left\{u \in H_{0}^{1}(\Omega): u \leq M\right.$ and $u$ concave $\}$. Thus, the associated Euler-Lagrange formulation (in terms of maximal monotone graphs) is given by

$$
-\operatorname{div}\left(F^{\prime}(|\nabla u|) \frac{\nabla u}{|\nabla u|}\right)+\beta(u) \ni 0 .
$$

In order to simplify the presentation for nonnegative functions $u(x)$, we notice that in the radial case and for nonincreasing functions $u=u(r), r=|x|$, we have

$$
-\operatorname{div}\left(F^{\prime}(|\nabla u|) \frac{\nabla u}{|\nabla u|}\right)=r\left(F^{\prime}\left(\left|u^{\prime}(r)\right|\right)\right)^{\prime} .
$$

In particular, we can identify the above Euler-Lagrange equation with formulation (11) by choosing

$$
A(t) t=F^{\prime}(t)=\frac{-2 t}{\left(1+t^{2}\right)^{2}}
$$

and so

$$
A(t)=\frac{-2}{\left(1+t^{2}\right)^{2}}
$$

(see [16], [11], [5]). It is easy to see that the function $A$ satisfies the assumptions of Case 1. In particular

$$
\lim _{a \rightarrow+\infty} A(a) \int_{\alpha}^{a} \frac{d \tau}{\tau A(\tau)}=\frac{1}{4} .
$$


Example 2. The function $A(t)=-t^{m-2} /\left(1+t^{m}\right)^{2}$, with $m \geq 1$, corresponds to other type of resistance forces already proposed by Newton.

Example 3. A function $A$ satisfying the conditions of Case 2 is, for instance, $A(t)=$ $-\left(\beta_{A}-t\right)^{\sigma}$ with $\sigma \in(0,1)$.

\section{Radially symmetric solutions}

This section is devoted to the study of radially symmetric solutions. Then 11 leads to the question: Given $M>0$, find $\rho \in(0, R)$ and $u$ satisfying

$$
\left\{\begin{array}{l}
-\frac{1}{r} \frac{d}{d r}\left(r A\left(\left|u^{\prime}\right|\right) u^{\prime}\right)+\beta(u) \ni 0 \quad \text { in }(0, R), \\
u(R)=0, \quad-u^{\prime}\left(R^{-}\right)>\alpha_{A}, \\
u(r)=M \quad \text { in }[0, \rho] \text { and } u(r)<M \quad \text { in }(\rho, R] .
\end{array}\right.
$$

Our study will mainly focus on the properties of the coincidence set:

Theorem 3.1. Let $R>0$ be given. Assume that A satisfies the conditions of Case 1 . Then, for every $M>0$, there exists $\rho_{M} \in(0, R)$ such that for any $m \in\left(\alpha_{A},+\infty\right)$ there exists a solution $u(r)=u(r ; m)$ of the obstacle problem satisfying

(i) $u \equiv M$ in $\left[0, \rho_{m}\right]$ for some $\rho_{m} \in\left[\rho_{M}, R\right)$,

(ii) $-u^{\prime}\left(\rho_{m}\right)=m$,

(iii) $u$ is strictly concave in $\left(\rho_{m}, R\right)$ and $u \in W^{1, \infty}(0, R)$.

In Case 2, for every $0<M \leq R \beta_{A}$ there exist $0<\rho_{M_{1}}<\rho_{M_{2}}<R$ such that for any $m \in\left(\alpha_{A}, \beta_{A}\right)$ there exists a solution $u(r)=u(r ; m)$ of the obstacle problem satisfying

(i) $u \equiv M$ in $\left[0, \rho_{m}\right]$ for some $\rho_{m} \in\left[\rho_{M_{1}}, \rho_{M_{2}}\right)$,

(ii) $-u^{\prime}\left(\rho_{m}\right)=m$,

(iii) $u$ is strictly concave in $\left(\rho_{m}, R\right)$ and $u \in W^{1, \infty}(0, R)$.

Finally, in both cases, the map $M \mapsto \rho_{M}$ is decreasing and convex.

Remark 3.1. In the Newton case, $\alpha_{A}=1 / \sqrt{3}$, and the solution of the obstacle problem which corresponds to a minimum of energy is associated with $m=1$; see [16] and [11].

In the following we will write $\alpha$ instead of $\alpha_{A}$ and $\beta$ instead of $\beta_{A}$ in order to simplify the notations. The equation we have to deal with is

$$
\frac{d}{d r}\left(r A\left(\left|u^{\prime}(r)\right|\right) u^{\prime}(r)\right)=0
$$

and thus $r \mapsto r A\left(\left|u^{\prime}(r)\right|\right) u^{\prime}(r)$ is constant. We look for solutions $u$ such that:

$u$ is defined in $[0, R], u(R)=0$ and $u(\rho)=M$ for some $\rho \in(0, R)$. 
The fact that $-u^{\prime}\left(R^{-}\right)>\alpha$ implies there exists $\delta>0$ such that

$$
-u^{\prime} \geq \alpha \quad \text { on }(R-\delta, R) \text { for some } \delta>0 .
$$

Developing 21] we then obtain

$$
A\left(\left|u^{\prime}(r)\right|\right) u^{\prime}(r)+r\left(A^{\prime}\left(\left|u^{\prime}(r)\right|\right)\left|u^{\prime}(r)\right|+A\left(\left|u^{\prime}(r)\right|\right)\right) u^{\prime \prime}(r)=0 .
$$

Employing (23) and (24) with (15) in Case 1 and (18) in Case 2 yields

$$
\left(A^{\prime}\left(\left|u^{\prime}(r)\right|\right)\left|u^{\prime}(r)\right|+A\left(\left|u^{\prime}(r)\right|\right)\right) u^{\prime \prime}(r) \leq 0 .
$$

Lemma 3.1. For every solution $u$ of 21 satisfying $0<u<M$, we have $-u^{\prime} \in(\alpha,+\infty)$ in Case 1 and $-u^{\prime} \in(\alpha, \beta)$ in Case 2.

Proof. Case 1. Since for $r$ in $(R-\delta, R)$ we have $-u^{\prime}(r) \in(\alpha,+\infty)$, and since $u^{\prime \prime}<0$, it follows that $-u^{\prime}$ is increasing in this neighborhood and the property will be satisfied as long as $-u^{\prime}$ does not reach the value $\alpha$. Let us denote by $\gamma$ the maximal value of $r$ in $(0, R)$ such that $-u^{\prime}=\alpha$, if it exists. Then $-u^{\prime} \in(\alpha,+\infty)$ for every $r \in(\gamma, R)$. But we also know that $u^{\prime}(\gamma)=-\alpha$, and then taking $r=\gamma$ in 24) leads to $A(\alpha) \alpha=0$. On the other hand, we know that $\alpha>0$ and from (15) that $A>0$, hence we get a contradiction.

Case 2. The proof is the same as the previous one, with $-u^{\prime}(r) \in(\alpha,+\infty)$ replaced by $-u^{\prime}(r) \in(\alpha, \beta)$ and the assumptions $(6)$ by $(17)$ and $(15)$ by $(18)$.

Now we will try to solve the equation 21 under the conditions 22) employing a parametric method as in [8]. From [21] we have

$$
r A\left(\left|u^{\prime}(r)\right|\right) u^{\prime}(r)=R A\left(\left|u^{\prime}(R)\right|\right) u^{\prime}(R) .
$$

Since the value of $u^{\prime}\left(R^{-}\right)$will play a crucial role, we introduce $a=-u^{\prime}\left(R^{-}\right)$.

As $u$ is concave, $a$ will be the maximal value of the function $-u^{\prime}(r)$ in the interval $(\rho, R)$. Now, the idea is to take a new parameter to solve the differential equation. We use $t=-u^{\prime}(r)$ and observe that, since $u$ is assumed to be concave decreasing, $t>0$ and $-u^{\prime}$ is bijective. The equality 26 can be written in the form

$$
-r A(t) t=-R A(a) a
$$

so that

$$
r=\frac{\operatorname{RaA}(a)}{t A(t)}=r(t) .
$$

We differentiate $u$ with respect to $t$ to obtain

$$
\frac{d}{d t} u(r)=\frac{d}{d t}(u \circ r(t))=u^{\prime}(r(t)) \frac{d r}{d t}=t \operatorname{RaA}(a) \frac{(t A(t))^{\prime}}{t^{2} A^{2}(t)} .
$$


Using the fact that $u(R)=0$, we then obtain

$$
\begin{aligned}
u(R)-u(t) & =\int_{t}^{a} \frac{d u}{d t} d \tau-u(t) \\
& =\operatorname{RaA}(a) \int_{t}^{a} \tau \frac{A(\tau)+\tau A^{\prime}(\tau)}{\tau^{2} A^{2}(\tau)} d \tau \\
& =\operatorname{RaA}(a) \int_{t}^{a}\left(\frac{A^{\prime}(\tau)}{A^{2}(\tau)}+\frac{1}{\tau A(\tau)}\right) d \tau \\
& =\operatorname{Ra}\left(\frac{A(a)}{A(t)}-1\right)+\operatorname{RaA}(a) \int_{t}^{a} \frac{d \tau}{\tau A(\tau)}
\end{aligned}
$$

That is,

$$
u(t)=R a-R a \frac{A(a)}{A(t)}-\operatorname{RaA}(a) \int_{t}^{a} \frac{d \tau}{\tau A(\tau)} .
$$

Thus, the problem can be rephrased as follows: let $\rho \in(0, R)$ and $M \geq 0$ be given. We want to find $a>\alpha$ such that there exists $t(a)$ with

$$
\rho t(a) A(t(a))=\operatorname{Ra} A(a)
$$

and

$$
M=R a-R a \frac{A(a)}{A(t)}-\operatorname{RaA}(a) \int_{t}^{a} \frac{d \tau}{\tau A(\tau)} .
$$

We have the following theorem:

Theorem 3.2. Let $M>0$ be a given number. In Case 1, there exists $\rho_{M}$ such that 29](30) admits a solution if $\rho$ belongs to $\left(\rho_{M}, R\right)$, and none if $\rho \in\left(0, \rho_{M}\right)$.

In Case 2, if $M>R \beta$ there is no solution and if $M \leq R \beta$ there exists a solution if and only if $\rho \in\left(\rho_{M},\left(R-\rho_{M}\right) \beta\right)$.

Remark 3.2. In Case 1 , if we consider $\rho \in\left(\rho_{M}, R\right)$ and look for solutions to (11) in the annulus $\Omega=(\rho, R)$, we see that the functional is convex, therefore the minimizer is unique, and since we have found a radial solution, it is the unique solution. The same happens to be true in Case 2 under suitable assumptions.

In order to prove the above theorem we will need the following two lemmas:

Lemma 3.2. The function $a \mapsto t(a)$ defined in $(29)$ is an increasing function from $\left(a_{\rho},+\infty\right)$ into $(\alpha,+\infty)$ in Case 1 , and from $\left(a_{\rho}, \beta\right)$ into $(\alpha, \beta)$ in Case 2 , where $a_{\rho}$ is the solution of $\rho \alpha A(\alpha)=R a_{\rho} A\left(a_{\rho}\right)$.

Remark 3.3. The existence of $a_{\rho}$ is ensured by the fact that $\alpha A(\alpha)$ is the minimum of the function $t A(t)$ (which is negative) and since $\rho<R$. 
Proof. Case 1. From the assumption (6) we know that the function $t \mapsto t A(t)$ is increasing on $(\alpha,+\infty)$ and then using Lemma 3.1, we obtain $t(a)<a$. Indeed, suppose not. Then $t(a) A(t(a)) \geq a A(a)$ with equality if and only if $t(a)=a$. This contradicts $\sqrt{29}$ if $t(a) \neq a$. In that last case 29] implies $\rho=R$, which is not possible since $u(\rho)=M>0$ and $u(R)=0$.

Now we differentiate 29 , with respect to $a$ to obtain

$$
\rho \frac{d t}{d a}\left(A(t(a))+A^{\prime}(t(a)) t(a)\right)=R\left(A(a)+a A^{\prime}(a)\right) .
$$

The two terms in brackets are positive by (6) and Lemma 3.1. so that $d t / d a>0$. Finally, we know that $t(a)$ has to be greater than $\alpha$, hence the minimal value for $a$ arises for $t(a)=\alpha$. Passing to the limit in 29] as $a$ tends to $+\infty$ leads to

$$
\lim _{a \rightarrow+\infty} \rho t(a) A(t(a))=0 .
$$

Therefore $\rho>0$ implies $\lim _{a \rightarrow+\infty} t(a)=+\infty$.

Case 2. The proof is the same as the previous one upon replacing $(\alpha,+\infty)$ by $(\alpha, \beta)$ and assumption (6) by (17). By passing to the limit in (29) as $a$ tends to $\beta$, we obtain

$$
\lim _{a \rightarrow \beta} \rho t(a) A(t(a))=0 .
$$

Then, since $\rho>0$, the only possibility is $\lim _{a \rightarrow \beta} t(a)=\beta$ in view of assumptions (17) and $(18)$.

Lemma 3.3. The function $a \mapsto u \circ t(a)$ defined by (30) is increasing and converges to $+\infty$ as a converges to $+\infty$ in Case 1. The function $a \mapsto u \circ t(a)$ defined by (30) is increasing and converges to $(R-\rho) \beta$ as a converges to $\beta$ in Case 2.

Proof. We have

$$
\frac{d}{d a} u \circ t(a)=\frac{d}{d a}\left(R a-R a \frac{A(a)}{A(t)}-\operatorname{RaA}(a) \int_{t}^{a} \frac{d \tau}{\tau A(\tau)}\right) .
$$

Using 29, we can express it as

$$
\begin{aligned}
\frac{d}{d a} u \circ t(a)= & \frac{d}{d a}\left(\operatorname{Ra}-\rho t(a)-\operatorname{RaA}(a) \int_{t(a)}^{a} \frac{d \tau}{\tau A(\tau)}\right) \\
= & R-\rho \frac{d t}{d a}-\operatorname{RA}(a) \int_{t(a)}^{a} \frac{d \tau}{\tau A(\tau)}-\operatorname{RaA}^{\prime}(a) \int_{t(a)}^{a} \frac{d \tau}{\tau A(\tau)} \\
& -\operatorname{RaA}(a)\left[\frac{1}{a A(a)}-\frac{1}{t(a) A(t(a))} \frac{d t}{d a}\right] \\
= & -R\left(A(a)+a A^{\prime}(a)\right) \int_{t(a)}^{a} \frac{d \tau}{\tau A(\tau)}
\end{aligned}
$$


Using Lemma 3.1 together with (6) in Case 1 and together with (17) in Case 2, we get

$$
\frac{d}{d a} u \circ t(a)>0 \text {. }
$$

Thus, if a solution of (30) exists it is unique.

Now, we split up the remainder of the proof into two cases.

Case 1. We have to compute

$$
\lim _{a \rightarrow+\infty}\left(\operatorname{Ra}-\rho t(a)-\operatorname{RaA}(a) \int_{t(a)}^{a} \frac{d \tau}{\tau A(\tau)}\right),
$$

and prove that it is equal to $+\infty$. Employing (6) we obtain

$$
R a-\rho t(a)-\operatorname{RaA}(a) \int_{t(a)}^{a} \frac{d \tau}{\tau A(\tau)} \geq(R-\rho) t(a)
$$

and since $\rho<R$ and $\lim _{a \rightarrow+\infty} t(a)=+\infty$, we find the result.

Case 2. Here, we have to compute

$$
\lim _{a \rightarrow \beta}\left(\operatorname{Ra}-\rho t(a)-\operatorname{RaA}(a) \int_{t(a)}^{a} \frac{d \tau}{\tau A(\tau)}\right) .
$$

From 29 we have $(R-\rho) a=R a-\rho t(a)-\frac{R a A(a)(a-t(a))}{t(a) A(t(a))}$. On the other hand, from (17) and (18) we get

$$
\begin{aligned}
R a-\rho t(a)-\frac{\operatorname{RaA}(a)(a-t(a))}{t(a) A(t(a))} & \geq \operatorname{Ra}-\rho t(a)-\operatorname{RaA}(a) \int_{t(a)}^{a} \frac{d \tau}{\tau A(\tau)} \\
& \geq(R-\rho) t(a) .
\end{aligned}
$$

Then since $\lim _{a \rightarrow \beta} t(a)=\beta$, we pass to the limit in the inequality to obtain the result.

Proof of Theorem 3.2. Step 1. We will now compute the minimal value of $u$. From Lemma 3.2 we know that the smallest admissible value for $a$ occurs when $t(a)=\alpha$; we denote this value by $a_{\rho}$. In that case we have

$$
u \circ t\left(a_{\rho}\right)=u(\alpha)=R a_{\rho}\left(1-\frac{A\left(a_{\rho}\right)}{A(\alpha)}-A\left(a_{\rho}\right) \int_{\alpha}^{a_{\rho}} \frac{d \tau}{\tau A(\tau)}\right)
$$

and using (36) we can express it as

$$
u(\alpha)=R a_{\rho}-\rho \alpha-\rho \alpha A(\alpha) \int_{\alpha}^{a_{\rho}} \frac{d \tau}{\tau A(\tau)} .
$$

This is the minimal value of $u$ since $u$ is increasing in $a$ and if $M<u(\alpha)$ there is no solution of 29-30 in both cases. Additionally, if $M>(R-\rho) \beta$, there is no solution in Case 2. 
Step 2. The next point is to find a $\rho$ such that 29- 30 has a solution. If we write

$$
\rho \alpha A(\alpha)=R a_{\rho} A\left(a_{\rho}\right)
$$

and

$$
\mu(\rho)=R a_{\rho}-\rho \alpha-\rho \alpha A(\alpha) \int_{\alpha}^{a_{\rho}} \frac{d \tau}{\tau A(\tau)}
$$

then for any given $\rho \in(0, R)$, there exists $\mu(\rho)>0$ such that the equation (30) can be uniquely solved in Case 1 for all $M \in[\mu(\rho),+\infty)$ and in Case 2 for all $M \in[\mu(\rho)$, $(R-\rho) \beta]$.

We will first prove that the function $\rho \mapsto \mu(\rho)$ defined by 37 is decreasing from $+\infty$ to 0 in Case 1 and from $R \beta$ to 0 in Case 2 . In both cases it is convex. To this end, we differentiate (37) with respect to $\rho$ to obtain

$$
\frac{d \mu(\rho)}{d \rho}=R \frac{d a_{\rho}}{d \rho}-\alpha-\alpha A(\alpha) \int_{\alpha}^{a_{\rho}} \frac{d \tau}{\tau A(\tau)}-\frac{\rho \alpha A(\alpha)}{a_{\rho} A\left(a_{\rho}\right)} \frac{d a_{\rho}}{d \rho} .
$$

Using (36) we get

$$
\frac{d \mu(\rho)}{d \rho}=-\alpha-\alpha A(\alpha) \int_{\alpha}^{a_{\rho}} \frac{d \tau}{\tau A(\tau)},
$$

which is negative by (15) in Case 1 or 18 in Case 2 . This allows us to establish that the map $M \mapsto \rho_{M}$ is decreasing.

We now distinguish two cases.

Case 1. Passing to the limit as $\rho$ tends to 0 in 36 leads to $a_{\rho}$ converging to $+\infty$. Combining (36) and (37) we obtain

$$
\mu(\rho)=R a_{\rho}-\rho \alpha-R a_{\rho} A\left(a_{\rho}\right) \int_{\alpha}^{a_{\rho}} \frac{d \tau}{\tau A(\tau)},
$$

or

$$
\mu(\rho)=-\rho \alpha+R a_{\rho}\left(1-A\left(a_{\rho}\right) \int_{\alpha}^{a_{\rho}} \frac{d \tau}{\tau A(\tau)}\right) .
$$

Then, thanks to [16, we obtain

$$
\lim _{a_{\rho} \rightarrow+\infty}\left(1-A\left(a_{\rho}\right) \int_{\alpha}^{a_{\rho}} \frac{d \tau}{\tau A(\tau)}\right)>0
$$

hence

$$
\lim _{a_{\rho} \rightarrow+\infty} \mu(\rho)=+\infty
$$

On the other hand, if $\rho$ converges to $R$, we deduce from $(36$ and the assumption (6) that

$$
\lim _{\rho \rightarrow R} a_{\rho}=\alpha,
$$

thus $\lim _{\rho \rightarrow R} \mu(\rho)=0$ by 37 . 
Case 2. Passing to the limit as $\rho$ tends to 0 in 36 leads to $a_{\rho} A\left(a_{\rho}\right)$ converging to 0 . Since $a_{\rho}>\alpha>0$, the only possibility is $a_{\rho} \rightarrow \beta$. Then passing to the limit in (37) yields

$$
\lim _{\rho \rightarrow 0} \mu(\rho)=R \beta-\lim _{\rho \rightarrow 0} \rho \alpha A(\alpha) \int_{\alpha}^{a_{\rho}} \frac{d \tau}{\tau A(\tau)} .
$$

Since

$$
\lim _{\rho \rightarrow 0} \int_{\alpha}^{a_{\rho}} \frac{d \tau}{\tau A(\tau)}=\int_{\alpha}^{\beta} \frac{d \tau}{\tau A(\tau)},
$$

which is finite under the assumption 19 , we obtain

$$
\lim _{\rho \rightarrow 0} \mu(\rho)=R \beta .
$$

We use (36) and the assumption (17) to get

$$
\lim _{\rho \rightarrow R} a_{\rho}=\alpha
$$

and thus from (37), we obtain $\lim _{\rho \rightarrow R} \mu(\rho)=0$.

In order to prove the convexity of $\mu$, we twice differentiate 37) with respect to $\rho$ and, employing 36, we see that

$$
\frac{d^{2} \mu(\rho)}{d \rho^{2}}=-\frac{\alpha A(\alpha)}{a_{\rho} A\left(a_{\rho}\right)} \frac{d a_{\rho}}{d \rho} .
$$

Differentiation of (36) with respect to $\rho$ yields

$$
\alpha A(\alpha)=R \frac{d a_{\rho}}{d \rho} \frac{d}{d t}(t A(t))_{t=a_{\rho}} .
$$

The assumption (6) in Case 1 or (17) in Case 2 guarantee that

$$
\frac{d a_{\rho}}{d \rho}<0
$$

and substituting this into (48) leads to

$$
\frac{d^{2} \mu(\rho)}{d \rho^{2}}>0
$$

This implies that the map $M \mapsto \rho_{M}$ is convex, finishing the proof of the theorem.

We will now deal with the property of minimum solutions. We set $\Omega=B(0, R)$ and consider the following problem associated to 10 :

$$
\operatorname{Min}_{K} \int_{\Omega} F(|\nabla u|) d x
$$

where $K=\left\{u \in H_{0}^{1}(\Omega): u\right.$ satisfies the assumptions of 10 and is radially symmetric $\}$. Notice that the formulation corresponding to the stationary points of the functional 52 leads to $A(|\nabla u|)=F^{\prime}(|\nabla u|) /|\nabla u|$. We have: 
Theorem 3.3. Let $M>0$ be given. Then there exists a unique value of $m \in\left(\alpha_{A},+\infty\right)$ in Case 1 , and $m \in\left(\alpha_{A}, \beta_{A}\right)$ in Case 2 , such that the solution $u(r ; m)$ given in Theorem 3.1 is minimal among all solutions given in that theorem.

Proof. Let $u_{\rho}$ be a solution to 20]. We have to compute

$$
E(\rho):=\int_{\rho}^{R} r F\left(u_{\rho}^{\prime}(r)\right) d r+\int_{0}^{\rho} r F\left(u_{\rho}^{\prime}(r)\right) d r
$$

which can be written as

$$
E(\rho)=\int_{\rho}^{R} r F\left(u_{\rho}^{\prime}(r)\right) d r+\frac{F(0)}{2} \rho^{2} .
$$

Since $E(\rho)$ cannot be expressed in a simple way as a function of $\rho$, we will use the former parameterization, that is, $t=-u^{\prime}(r)$ and

$$
-r A(t) t=-R A(a) a
$$

so that

$$
r=\frac{\operatorname{RaA}(a)}{t A(t)}=r(t)
$$

Integration by parts leads to

$$
\begin{aligned}
E(\rho)=\Psi(T, a) & =-R^{2} \int_{T}^{a} \frac{F^{\prime}(a)^{2} F^{\prime \prime}(\tau) F(\tau)}{F^{\prime}(\tau)^{3}} d \tau \\
& =\frac{R^{2}}{2}\left(F(a)-\frac{F(T) F^{\prime}(a)^{2}}{F^{\prime}(T)^{2}}-F^{\prime}(a)^{2} \int_{T}^{a} \frac{d \tau}{F^{\prime}(\tau)}+\frac{F(0)}{F^{\prime}(T)^{2}}\right) .
\end{aligned}
$$

The function $\Psi(T, a)$ has to be minimized under the constraint 30 , that is,

$$
M=R a-R T \frac{F^{\prime}(a)}{F^{\prime}(T)}-R F^{\prime}(a) \int_{T}^{a} \frac{d \tau}{F(\tau)}:=\Xi(T, a) .
$$

A careful computation shows

$$
\begin{aligned}
\frac{\partial}{\partial T} \Psi(T, a) & =R^{2} \frac{F^{\prime}(a)^{2}}{F^{\prime}(T)^{3}} F^{\prime \prime}(T)(F(T)-F(0)) \\
\frac{\partial}{\partial a} \Psi(T, a) & =-R^{2} \frac{F^{\prime}(a)}{F^{\prime}(T)^{2}} F^{\prime \prime}(a)\left(F(T)+F^{\prime}(T)^{2} \int_{T}^{a} \frac{d \tau}{F^{\prime}(\tau)}-F(0)\right), \\
\frac{\partial}{\partial T} \Xi(T, a) & =R \frac{F^{\prime}(a)}{F^{\prime}(T)^{2}} F^{\prime \prime}(T) T \\
\frac{\partial}{\partial a} \Xi(T, a) & =-R \frac{1}{F^{\prime}(T)} F^{\prime \prime}(a)\left(T+F^{\prime}(T) \int_{T}^{a} \frac{d \tau}{F^{\prime}(\tau)}\right) .
\end{aligned}
$$


Therefore, the Lagrange condition $\frac{\partial \Psi}{\partial a} \frac{\partial \Xi}{\partial T}=\frac{\partial \Psi}{\partial T} \frac{\partial \Xi}{\partial a}$ reduces to

$$
\left(F(T)+F^{\prime}(T)^{2} \int_{T}^{a} \frac{d \tau}{F^{\prime}(\tau)}-F(0)\right) T=(F(T)-F(0))\left(T+F^{\prime}(T) \int_{T}^{a} \frac{d \tau}{F^{\prime}(\tau)}\right)
$$

since the other terms do not vanish. This, again, can be simplified as

$$
F(0)=F(T)-F^{\prime}(T) T
$$

which can be written in terms of $A$ as

$$
\int_{0}^{T} \tau A(\tau) d \tau=T^{2} A(T)
$$

Set $g(t)=\int_{0}^{t} \tau A(\tau) d \tau-t^{2} A(t)$. Thus,

$$
g^{\prime}(t)=-t(t A(t))^{\prime}
$$

and hence $g$ is increasing from 0 to $g\left(\alpha_{a}\right)$ and then decreasing (we used 6 in Case 1 or (17) in Case 2). In particular, the equation (56) has at most one solution in $\left(\alpha_{A},+\infty\right)$ in Case 1 or in $\left(\alpha_{A}, \beta_{A}\right)$ in Case 2. On the other hand, employing (6) (or (17)) we have

$$
\int_{\alpha_{A}}^{t} \tau A(\tau) d \tau \leq t A(t)\left(t-\alpha_{A}\right) \quad \text { for } t>\alpha_{A}\left(\text { or } t \in\left(\alpha_{A}, \beta_{A}\right)\right)
$$

Hence

$$
g(t) \leq \int_{0}^{\alpha_{A}} \tau A(\tau) d \tau+t A(t)\left(t-\alpha_{A}\right) .
$$

Now thanks to 15 in Case 1 ,

$$
\lim _{t \rightarrow+\infty} g(t) \leq \int_{0}^{\alpha_{A}} \tau A(\tau) d \tau<0
$$

and using 18 in Case 2,

$$
\lim _{t \rightarrow \beta_{A}} g(t) \leq \int_{0}^{\alpha_{A}} \tau A(\tau) d \tau<0
$$

This implies the uniqueness of the value of $T$ satisfying [55, which is called $m$ in the theorem, and it concludes the proof. 


\section{On the flat region for the non-radially symmetric case}

In this section we give a partial answer to the following question raised by Buttazzo and Kawohl ([6]): let $u$ be a solution of the Newton problem of minimal resistance. Is there always a flat region for such a solution, i.e. does there exist an open set $\Omega_{0} \subset \Omega$ such that $u \equiv M$ on $\Omega_{0}$ ?

The results obtained in the previous section allow us to give the following answer:

Theorem 4.1. Let $A$ and $\beta$ be as in the previous sections. Let $u \in H_{0}^{1}(\Omega)$ be such that

$$
\left\{\begin{array}{l}
-\operatorname{div}(A(|\nabla u|) \nabla u)+\beta(u) \ni 0 \quad \text { in } \Omega \\
0 \leq u(x) \leq M \quad \text { for } x \in \Omega
\end{array}\right.
$$

Assume that

$$
\begin{gathered}
\qquad|\nabla u| \geq \alpha_{A} \quad \text { if } u<M, \\
\left\{\begin{array}{r}
\text { there exist } x_{0} \in \Omega \text { and } R>0 \text { such that } \\
-A(|\nabla u(x)|) \nabla u(x) \cdot \frac{x-x_{0}}{\left|x-x_{0}\right|} \leq A(a) a \\
\text { for some } a \geq \alpha_{A} \text { and a.e. } x \in B\left(x_{0}, R\right), \text { where } u(x)<M .
\end{array}\right.
\end{gathered}
$$

Then there exists $\rho_{a} \in(0, R)$ such that $u(x)=M$ for a.e. $x \in B\left(x_{0}, \rho_{a}\right)$.

In order to prove this we need the following result:

Lemma 4.1. Let $u$ be as before, and let $u_{a}(x)=u\left(\left|x-x_{0}\right| ; a\right)$ be the radially symmetric solution of the obstacle problem given in Theorem 3.1 (i.e., such that $u_{a}(x)=M$ for a.e. $\left.x \in B\left(x_{0}, \rho_{a}\right)\right)$. Define

$$
\Omega_{x_{0}, R, \rho_{a}}=\left\{x \in B\left(x_{0}, R\right) \backslash B\left(x_{0}, \rho_{a}\right): u(x)<M\right\} .
$$

Then $u_{a}(x) \leq u(x)$ for a.e. $x \in \Omega_{x_{0}, R, \rho_{a}}$.

Proof of Theorem 4.1. Since $u(x) \leq M$, we deduce from the comparison stated in the previous lemma that, in particular, $u(x)=M$ on $B\left(x_{0}, \rho_{a}\right)$.

Proof of Lemma 4.1. Taking $\left[u_{a}(x)-u(x)\right]^{+}$as test function in the definitions of weak solution of the problems satisfied by $u_{a}$ and $u$, and subtracting both expressions we arrive at

$$
\begin{array}{r}
\int_{\Omega_{x_{0}, R, \rho_{a}}}\left(A\left(\left|\nabla u_{a}\right|\right) \nabla u_{a}-A(|\nabla u|) \nabla u\right) \cdot \nabla\left[u_{a}-u\right]^{+} d x+\int_{\Omega_{x_{0}, R, \rho_{a}}}\left(b_{a}-b\right)\left[u_{a}-u\right]^{+} d x \\
=\int_{\partial \Omega_{x_{0}, R, \rho_{a}}}\left[u_{a}-u\right]^{+}\left(A\left(\left|\nabla u_{a}\right|\right) \nabla u_{a}-A(|\nabla u|) \nabla u\right) \cdot \mathbf{n} d x:=I,
\end{array}
$$


where $b \in L^{1}(\Omega), b_{a} \in L^{1}\left(B\left(x_{0}, R\right)\right)$, and $b(x) \in \beta(u(x)), b_{a}(x) \in \beta\left(u_{a}(x)\right)$ for a.e. $x \in \Omega$ and $B\left(x_{0}, R\right)$ respectively. Write $\partial \Omega_{x_{0}, R, \rho_{a}}=\Gamma_{R} \cup \Gamma_{M} \cup \Gamma_{\rho_{a}}$, where

$$
\begin{aligned}
\Gamma_{R} & :=\left\{x \in \partial \Omega_{x_{0}, R, \rho_{a}}:\left|x-x_{0}\right|=R\right\}, \\
\Gamma_{\rho_{a}} & :=\left\{x \in \partial \Omega_{x_{0}, R, \rho_{a}}:\left|x-x_{0}\right|=\rho_{a}\right\}, \\
\Gamma_{M} & :=\left\{x \in \partial \Omega_{x_{0}, R, \rho_{a}}: u(x)=M\right\},
\end{aligned}
$$

we deduce that $I \geq 0$ (recall that $\beta$ is a monotone graph and that, since the function $t \mapsto A(t) t$ is nondecreasing, the vectorial function $\mathbf{v} \mapsto A(|\mathbf{v}|) \mathbf{v}$ is a monotone operator from $\mathbb{R}^{N}$ to $\mathbb{R}^{N}$ ). But

$$
\int_{\Gamma_{R} \cup \Gamma_{M}}\left[u_{a}-u\right]^{+}\left(A\left(\left|\nabla u_{a}\right|\right) \nabla u_{a}-A(|\nabla u|) \nabla u\right) \cdot \mathbf{n} d x=0
$$

because $\left[u_{a}-u\right]^{+}=0$ there, and thus

$$
\int_{\Gamma_{\rho_{a}}}\left[u_{a}-u\right]^{+}\left(A\left(\left|\nabla u_{a}\right|\right) \nabla u_{a}-A(|\nabla u|) \nabla u\right) \cdot \mathbf{n} d x \geq 0 .
$$

On $\Gamma_{\rho_{a}}, \mathbf{n}=x-x_{0} /\left|x-x_{0}\right|$ and so, since $\left|\nabla u_{a}(x)\right|=-\frac{d}{d r} u_{a}\left(\rho_{a}\right)=a$ if $x \in \Gamma_{\rho_{a}}$, by using the assumption (59), we arrive at

$$
\left(A\left(\left|\nabla u_{a}\right|\right) \nabla u_{a}-A(|\nabla u|) \nabla u\right) \cdot \mathbf{n} \leq 0 \quad \text { on } \Gamma_{\rho_{a}} .
$$

Then

$$
\int_{\Omega_{x_{0}, R, \rho_{a}}}\left(A\left(\left|\nabla u_{a}\right|\right) \nabla u_{a}-A(|\nabla u|) \nabla u\right) \cdot \nabla\left[u_{a}-u\right]^{+} d x=0,
$$

which, by the strict monotonicity of $A$ on this range of values, implies the conclusion.

\section{References}

[1] Armanini, E.: Sulla superficie di minima resistenza. Ann. Mat. Pura Appl. (3) 4, 131-149 (1900) JFM 31.0709.02

[2] Botteron, B., Marcellini, P.: A general approach to the existence of minimizers of onedimensional non-coercive integrals of the calculus of variations. Ann. Inst. H. Poincaré 8, 197-223 (1991) Zbl 0729.49002 MR 1096604

[3] Brezis, H.: Opérateurs maximaux monotones et semigroupes de contractions dans les espaces de Hilbert. North-Holland, Amsterdam (1973) Zbl 0252.47055 MR 0348562

[4] Brezis, H., Kinderlehrer, D.: The smoothness of solutions to nonlinear variational inequalities. Indiana Univ. Math. J. 23, 831-844 (1974) Zbl 0278.49011 MR 0361436

[5] Buttazzo, G., Ferone, V., Kawohl, B.: Minimum problems over sets of concave functions and related questions. Math. Nachr. 173, 71-89 (1993) Zbl 0835.49001 MR 1336954

[6] Buttazzo, G., Kawohl, B.: On Newton's problem of minimal resistance. Math. Intelligencer 15, 7-12 (1993) Zbl 0800.49038 MR 1240664

[7] Comte, M., Díaz, J. I.: Paper in preparation 
[8] Comte, M., Lachand-Robert, T.: Newton's problem of the body of minimal resistance under a single-impact assumption. Calc. Var. 12, 173-211 (2001) Zbl 0998.49012 MR 1821236

[9] Eggers Jr., A. J., Resnikoff, M. M., Dennis, D. H.: Bodies of revolution having minimum drag at high supersonic airspeeds. NASA Report 1306 (1958)

[10] Euler, L.: Principes généraux du mouvement des fluides. Mémoires de l’Académie des Sciences de Berlin 11, 217-273 (1755)

[11] Goldstine, H. H.: A History of the Calculus of Variations from the 17th through the 19th Century. Springer, Heidelberg (1980) Zbl 0452.49002 MR 0601774

[12] Guasoni, P.: Problemi di ottimizzazione di forma su classi di insiemi convessi. Laurea Thesis, Univ. of Pisa (1996)

[13] Kinderlehrer, D., Stampacchia, G.: An Introduction to Variational Inequalities and Their Applications. Academic Press, New York (1990) Zbl 0457.35001 MR 1786735

[14] Lachand-Robert, T., Oudet, É.: Minimizing within convex bodies using a convex hull method. SIAM J. Optim., to appear

[15] Miele, A.: Theory of Optimum Aerodynamic Shapes. Academic Press, London (1965) Zbl 0265.76076

[16] Newton, I.: Philosophiae Naturalis Principia Mathematica. (1686)

[17] Wagner, A.: A remark on Newton's resistance formula. Z. Angew. Math. Mech. ZAMM 79, 423-427 (1999) Zbl 0926.76101 MR 1690760 